\title{
A METHOD FOR THE INVESTIGATION OF GLYPH SYNTAX
}

\author{
By Nicholas A. Hopkins, \\ University of Texas
}

\section{SUMMARY}

This paper will outline a method for the investigation of the syntax of the Mayan hieroglyphs which bypasess many of the problems previously encountered in attempts at decipherment. Decipherment has proved difficult largely because it required competence in 1) Mayan epigraphy, 2) the Mayan language, and 3) techniques by which the first could be correlated with the second. Furthermore, most previous attempts at decipherment have been based on a prior assumption of the relation of the glyphs to the language spoken by their writers -that the glyphs form an alphabet, that they form a syllabary, that they are ideographic, etc. In addition, the assumption has often been made that some glyphs can be positively identified as having a certain phonetic, syllabic, or ideographic value. It should be clear that making the wrong assumption at the beginning of a study can only lead to compounded errors in the later stages. Neither type of assumption, however, is necessary for the beginnings of decipherment, and in fact both types of assumption are ill-advised before considerably more is known about the internal structure of the glyphic system than is presently known. The present method attempts to bypass these typical failures of prior investigators.

\section{ASSUMPTIONS}

Three assumptions underlie this method: 1) the Mayan hieroglyphs are based on a Mayan language spoken in Yucatán and adjacent areas at the time the glyphic system was being devised; 2) the syntactic structure of the glyphic system can be discovered by the examination of a large number of short, graphically dicrete, strings of glyphic elements; 3) the syntactic structures of both the glyphs and the language should be similar if not identical. 
The first assumption seems justified, as the known distribution of hieroglyphic inscriptions closely matches an area which can safely be said to have been occupied by speakers of a language ancestral to modern Yucatec Maya in the first century A.D. The second and third assumptions are no more than a repetition of the theories of modern structural linguistics.

The syntactic structure of the glyphs appears to be a reasonable point of departure for decipherment. It is not known, nor can it be safely assumed, that the Mayan writing system was either an alphabet, a syllabary, an ideographic system, or a mixture of two or more of these types of writing systems. What all these types of writing systems have in common, however, is that all are tied to the syntactic structure of the language. That is, whether the units recorded are phones, phonemes, groups of phonemes, morphemes, phrases, or whatever, the units are recorded in the order in which they occur in the spoken language. This is a common, if not universal, characteristic of writing systems, but this point seems to have been overlooked in the study of Mayan hieroglyphs. Its implication for decipherment is this: the syntactic structure of the glyphs may be studied without prior assumption of the nature of Mayan writing, and in fact a much more educated guess as to the nature of that system may be made after the syntactic structure of the glyphs has been determined.

Mayan languages in general, and Yucatec in particular, are characterized by a root inventory almost entirely composed of mono-syllabic roots of the shape CVC and affixes of other, shorter, shapes. The selection of consonants which may occur in a given root is limited. In sequences of roots, or sequences of a root plus affixes, many combinations of phonemes may not occur but are replaced by other combinations of phonemes. On the level of morphemes, Mayan languages characteristically have two large form classes; nouns and verbs, each of which has major subdivisions. Roots with initial glottal stops take a different set of prefixes from other roots. Intransitive verb roots take one set of prefixes, transitive verb roots take another set. Verbal affixes are in the main different from nominal affixes. A number of suffixes occur which form noun stems from verb roots, verb stems from noun roots, intransitive verb stems from transitive verb roots, and so on. That is, there are several major form classes of roots, each of which occurs in different environments which can be defined syntactically. Likewise there are phonological changes which take place on roots and affixes in syntactically-defined phonological environment.

The syntax of glyphs should lend itself to discovery by presentlyexisting linguistic techniques, as the discovery procedure is nothing 
more than a description of which sequences of glyphs may occur, which glyphs fall into the same co-occurrence classes, and which classes of glyphs occur in which orders. The available texts give ample data for an adequate study of glyph syntax. Given a description of the syntax of glyphs, it should be possible to compare the peculiarities of the glyph system with the peculiarities of the language of its inventors, and so determine the nature of the writing system. At the very least, a thorough study of glyph syntax cannot fail to make a more educated guess possible. It is already clear that certain glyphs occur only as "main signs", others occur only as "prefixes", other only as "suffixes", and still others as more than one of these types. The study of the internal structure of the glyphic system should make it possible to determine whether the co-occurrence restrictions more closely resemble the restrictions of phonemic or morphemic units of the language.

I propose to carry out this investigation by the following means. First, to avoid the necessity to become an expert Mayan epigrapher, I propose to use Thompson's Catalog of Mayan Glyphs as a source of texts. All, or virtually all, legible inscriptions are recorded in this catalog, the glyph elements identified by numbers. The entries in the catalog are strings of no more than seven glyph elements which occur in the same cartouche; that is, they occur in strings graphically separated from other clusters of glyphs. That these blocks of glyphs do form structural entities is shown not only by the structure of the calendric inscriptions, but by the fact that some glyph elements occur only initially, others only finally, and so on. These are not arbitrary divisions of text, but have structural reality. They do not, of course, form units of text entirely unrelated to preceding and following units, but are isolated enough to form the starting point of an investigation.

Second, in the absence of a fully-reconstructed Proto-Mayan or Proto-Yucatec which would represent the language of the first century A.D., I propose to use as a language source modern Yucatec, tempered both by available information on Sixteenth-Century Yucatec and by tentative reconstructions of Proto-Mayan based on data from several Mayan languages. The state of Mayan reconstructions has advanced considerably in the last ten years, and good guesses as to the nature of early Yucatec can be made on the basis of the work carried out by Professors Norman A. McQuown and Terrence S. Kaufman. In any case, the language represented in the glyphs is probably some ancestor of modern Yucatec, and considering the nature of Mayan languages in general, cannot have been radically different from modern Yucatec.

Third, for the investigation of the syntax of glyphs, I propose to use high-speed modern computers for the isolation of glyph co-occur- 
rence classes. The first step in the study will be a compilation of a glyph concordance, listing all the sequences in which a given glyph occurs. This listing will be used as a tool for the isolation of co-occurrence classes. A preliminary study carried out in 1962 shows that many sequences of "prefixes" and "suffixes" in Thompson's Catalog are common, others infrequent. It is possible by purely mechanical means to compile a listing of all "main signs" which occur after the same sequences of "prefixes" and before the same "suffixes." This in essence defines form classes on the basis of mutual substitutability in identical or similar environments. The same procedure will define classes and sub-classes of affixes. The procedure is crude in that many glyphs will be impossible to class, due to infrequent or unique occurrence. The advantage lies, however, in the fact that the procedure is simple and easily programmed, can be carried out entirely by computer, and thus can be quickly accomplished.

Following the establishment of classes of glyphs will come the comparison of the structure of the glyphs with the phonological and morphological structures of the language to determine, or guess at, the nature of the writing system. Depending on the nature of the writing system this may be either a simple or a very involved stage of investigation. If, for instance, the glyphic writing records morphemes, the following situation should be true: Transitive verbs will form a set of glyphs grouped into a single form class which, when suffixed by a certain set of glyphs, may be preceded by a certain set of prefixes. When not so suffixed, however, they may only be preceded by another set of prefixes:

$$
\begin{aligned}
& \text { Prefix set A - Transitive root } \\
& \text { Prefix set B - Transitive root }- \text { Suffix. }
\end{aligned}
$$

That is, the suffixes are those which transform transitive verb roots into intransitive verb stems, calling for a different set of inflectional prefixes. This sort of co-occurrence relationship - that between Prefix set B and the Suffixes - should be relatively easy to interpret. Other relationships may be extremely difficult to interpret.

Reversing the procedure so that the form class of the language may be determined from the distributional peculiarities of classes of glyphs will require considerable insight on the part of the investigator. It may be that the nature of the writing system will not be easily discerned even after the study of glyph syntax is complete. One advantage of the procedure, however, is that it will give us much more data on which to base our hypotheses than is presently available, 
and a hypothesis based on data is far more valuable than one based on prior assumptions.

I propose, then, to carry out a study in which, with no prior assumptions concerning the nature of the Maya writing system and accepting no "known" values for individual glyphs, a study of the internal structure of cartouches is made. If we are fortunate, this study will allow us both to identify the nature of Maya writing and to correlate distributional peculiarities of glyphs with distributional peculiarities of phonemes or morphemes in the language spoken by the writers. The value of this result for further studies in decipherment is considerable. If we are not so fortunate as to succeed, however, the study will still serve as a check on other attempts at decipherment, as any proposed solution to the Mayan glyphs which does not satisfactorily account for the glyph classes and the sequences in which they occur cannot be correct. 